\title{
The Knack and Pitfall of the Retropancreatic Nerve Plexus Hanging Maneuver for Pancreatoduodenectomy for Pancreatic Head Carcinoma.
}

\section{Naokazu Chiba ( $\sim$ nchiba0632@yahoo.co.jp)}

Tokyo Medical University Hachioji Medical Center: Tokyo Ika Daigaku Hachioji Iryo Center https://orcid.org/0000-0002-1846-5987

\section{Shigeto Ochiai}

Tokyo Medical University Hachioji Medical Center: Tokyo Ika Daigaku Hachioji Iryo Center Takahiro Gunji

Tokyo Medical University Hachioji Medical Center: Tokyo Ika Daigaku Hachioji Iryo Center Toshimichi Kobayashi

Tokyo Medical University Hachioji Medical Center: Tokyo Ika Daigaku Hachioji Iryo Center

\section{Kosuke Hikita}

Tokyo Medical University Hachioji Medical Center: Tokyo Ika Daigaku Hachioji Iryo Center

\section{Toru Sano}

Tokyo Medical University Hachioji Medical Center: Tokyo Ika Daigaku Hachioji Iryo Center

\section{Koichi Tomita}

Tokyo Medical University Hachioji Medical Center: Tokyo Ika Daigaku Hachioji Iryo Center

\section{Shigeyuki Kawachi}

Tokyo Medical University Hachioji Medical Center: Tokyo lka Daigaku Hachioji Iryo Center

\section{Technical advance}

Keywords: Pancreatoduodenectomy, Retropancreatic nerve plexus, Hanging maneuver

Posted Date: October 16th, 2020

DOl: https://doi.org/10.21203/rs.3.rs-75488/v1

License: (c) (1) This work is licensed under a Creative Commons Attribution 4.0 International License. Read Full License 


\section{Abstract}

\section{Background}

The efficacy of the hanging maneuver for the retropancreatic nerve plexus (RNP) to enhance the confirmation of the margin status and to facilitate en-bloc resection for pancreatoduodenectomy (PD). In this report, we present the knack and pitfall of the hanging maneuver of the RNP.

\section{Methods}

The exit of the hanging maneuver of the RNP is the left part of the superior mesenteric artery (SMA), and the entry is the cranial part of the celiac axis. The entry of the hanging maneuver was connected to the dissection line on the right side of the celiac axis. Thereafter, the tape of the hanging maneuver was pulled to the right side, and the RNP was deployed widely. Finally, the RNP was easily dissected using a sealing device other than IPDA

\section{Results}

It is important to clarify the entrance and exit of the hanging taping in this procedure. This permitted the wide spaces between the SMA, SMV, and the resected side, and it was easier to identify the IPDA. By traction of the hanging maneuver tape, a clear line may be drawn between the resection side and the remaining side.

\section{Conclusions}

With the correct implementation of the hanging maneuver, we believe that it would be possible to obtain reliable $\mathrm{R} 0$ resection as well as a reduction in blood loss and operation time.

\section{Background}

Pancreatoduodenectomy (PD) is the only curative treatment for ductal adenocarcinoma of the pancreatic head. Tumors arising from the pancreatic head tend to spread toward the superior mesenteric artery (SMA) [1], and consequently, the margin surrounding the SMA is important for curative resection [2]. The most technically difficult part of PD is the resection of the retropancreatic nerve plexus on the right side of the SMA.

Recently, various studies have reported the efficacy of the hanging maneuver for the retropancreatic nerve plexus (RNP) to enhance the confirmation of the margin status and to facilitate en-bloc resection $[3,4]$. Originally, the hanging maneuver was reported by Belghiti et al. with respect to liver surgery, and was performed using a tape that was passed behind the liver to guide hepatic resection via the anterior approach [5].

Here, we present our hanging maneuver of the RNP, and the knack and pitfall of this procedure. 


\section{Results}

\section{Surgical technique (Video 1)}

\section{Artery first approach}

Depending on the degree of invasion around the SMA and superior mesenteric vein (SMV), the root of the middle colonic artery (MCA) is identified from the anterior surface of the transverse mesocolon and the SMA trunk is secured. As in preoperative three-dimensional (3D) simulation, the $\mathrm{J} 1$ artery and $\mathrm{J} 2$ artery, which arise from the MCA cranially, were ligated and divided. After taping the SMV trunk, the J1 vein arising from the SMV was ligated and divided. The left part of the SMA in this procedure is the exit of the hanging maneuver of the RNP (Figure 1A).

\section{Kocherization of the duodenum}

Dissection for kocherization was initiated at the outer edge of the duodenum and the pancreatic head was retracted to the left. The dissection layer was advanced to the left edge of the aorta to identify the root of the SMA and celiac axis. The cranial part of the celiac axis in this procedure was the entry of the hanging maneuver of the RNP (Figure 1B).

Thereafter, dissection of the hepatoduodenal ligament was performed as the conventional method and the bile duct, duodenum, and jejunum were dissected. The entry of the hanging maneuver was connected to the dissection line on the right side of the celiac axis (Figure 1C).

\section{Dissection of retropancreatic nerve plexus}

The pancreatic parenchyma was dissected after pulling the jejunum through the back of the SMA and the SMV to the right side. The hanging tape was passed from the entrance to the exit, as described. The exit of the hanging maneuver could then be modified to subtract the SMA and SMV, and the entry could be modified to subtract the portal vein (PV) (Figure 1D). The tape of the hanging maneuver was pulled to the right side, and the RNP was deployed widely (Figure 1E). In this case, according to the preoperative 3D simulation, there was only one inferior pancreaticoduodenal artery (IPDA). Thus, while pulling the hanging tape, the RNP was dissected using a sealing device other than IPDA and inferior pancreaticoduodenal vein (IPDV) (Figure 1F).

\section{Discussion}

It is very important to perform the hanging maneuver of the RNP to ensure the entry and exit of the hanging tape and to keep kocherization to the left side of the aorta. Therefore, it is important to understand the branching of the vascular vessels by performing a preoperative 3D simulation. With the 
correct implementation of the hanging maneuver, we believe that it would be possible to obtain reliable $\mathrm{R} 0$ resection as well as a reduction in blood loss and operation time.

There are a few similar existing reports on the hanging maneuver of the retropancreatic nerve plexus $[3,4$, $6,7]$. Although these reports have many discussions regarding the order for the hanging maneuver of the $\mathrm{RNP}$, it is important to clarify the entrance and exit of the hanging taping in this procedure. In open surgery, because of the ventral approach, it is desirable to pull the hanging tape after dissection of the duodenum and pancreatic parenchyma. Since laparoscopic surgery uses a caudal approach, we believe that this procedure may be useful before dissection of the duodenum or pancreatic parenchyma.

In this case, the hanging maneuver of the retropancreatic nerve plexus was performed after dividing the duodenum and pancreatic parenchyma. This permitted the wide spaces between the SMA, SMV, and the resected side, and it was easier to identify the IPDA. Moreover, since the branching of the IPDA was identified during preoperative 3D simulation, the traction of the hanging maneuver tape was very useful in reducing the operation time and blood loss after dividing the IPDA.

Finally, the hanging maneuver of the retropancreatic nerve plexus enabled reliable R0 resection. Due to the anatomical features of the SMA, SMV, and retropancreatic nerve plexus, the reliable excision line always appears as an oblique line in computed tomography (CT) (Fig. 2). In the ventral approach to laparotomy, the cutting direction is diagonally inward unless the hanging maneuver is performed. Therefore, resection of the retroperitoneal plexus is the most difficult procedure in PD. However, it is thought that the diagonal excision line can be facilitated by securing the entrance and exit of the hanging maneuver and performing sufficient kocherization. By traction of the hanging maneuver tape, a clear line may be drawn between the resection side and the remaining side. However, whether the artery-first approach improves $\mathrm{R} 0$ resection rate and contributes to survival benefit remains unclear [8]. In addition, whether resection using this procedure produces an oncological benefit remains unclear and requires further study.

\section{Declarations}

\section{Ethics approval and consent to participate}

This research had been approved by Tokyo Medical University Hachioji Medical Center Ethics Committee, and had consent to participate by written paper.

\section{Consent for publication}

All authors had consent for publication of this research.

\section{Availability of data and materials}


This research had been available for data and materials.

\section{Competing interests}

All authors declare no conflicts of interest.

\section{Funding}

Not applicable.

\section{Author's contributions}

Study design: Author NC, and SK

Acquisition of data: Author NC

Operation procedures were performed All authors

Analysis and interpretation: Author NC

Manuscript drafted by Author NC

Revision: Author N Chiba and SK

\section{Acknowledgements}

Not applicable.

\section{References}

1. Makino I, Kitagawa H, Ohta T, Nakagawara H, Tajima H, Ohnishi I, et al. Nerve plexus invasion in pancreatic cancer: spread patterns on histopathologic and embryological analyses. Pancreas 2008;37:358-65.

2. Noto $M$, Miwa $K$, Kitagawa $H$, Kayahara $M$, Takamura $H$, Shimizu $K$, et al. Pancreas head carcinoma: frequency of invasion to soft tissue adherent to the superior mesenteric artery. Am J Surg Pathol. 2005;29(8):1056-61.

3. Wakabayashi T, Tanaka K, Oyama H. A New Artery-First Approach for Pancreatoduodenectomy Using a Retropancreatic Nerve Plexus Hanging Maneuver Before Division of the Gastric Antrum and Pancreatic Parenchyma. J Gastrointest Surg. 2020. doi: 10.1007/s11605-020-04672-w. 
4. Mizuno S, Isaji S, Tanemura A, Kishiwada M, Murata A, Azumi A, et al. Anterior Approach to the Superior Mesenteric Artery by Using Nerve Plexus Hanging Maneuver for Borderline Resectable Pancreatic Head Carcinoma. J Gastrointest Surg (2014) 18:1209-1215.

5. Belghiti J, Guevara OA, Noun R. Liver hanging maneuver: a safe approach to right hepatectomy without liver mobilization. J Am Coll Surg. 2001;193:109-11.

6. Rotellar F, Pardo F, Benito A, Martı-Cruchaga P, Zozaya G, Cienfuegos JA. Laparoscopic resection of the uncinate process of the pancreas: the inframesocolic approach and hanging maneuver of the mesenteric root. Surg Endosc (2011) 25:3426-3427.

7. Kuroki T, Tajima Y, Kitasato A, Adachi T, Kanematsu T. Pancreas-hanging maneuver in laparoscopic pancreaticoduodenectomy: a new technique for the safe resection of the pancreas head. Surg Endosc (2010) 24:1781-1783.

8. Sabater L, Cugat E, Serrablo A, Suarez-Artacho G, Diez-Valladares L, Santoyo-Santoyo J, et al. Does the Artery-first Approach Improve the Rate of R0 Resection in Pancreatoduodenectomy? A Multicenter, Randomized, Controlled Trial. Ann Surg. 2019 Nov;270(5):738-746.

\section{Figures}

A:
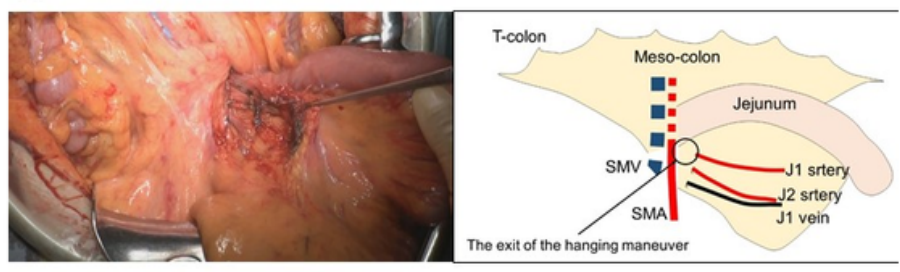

B:
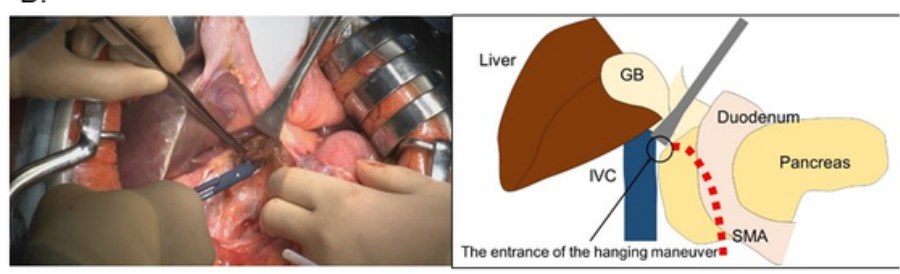

C:

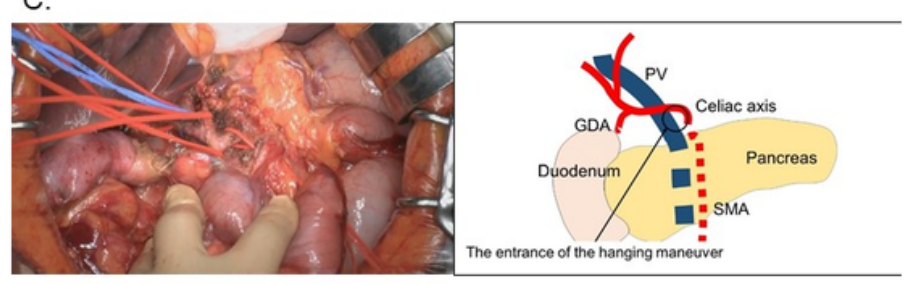

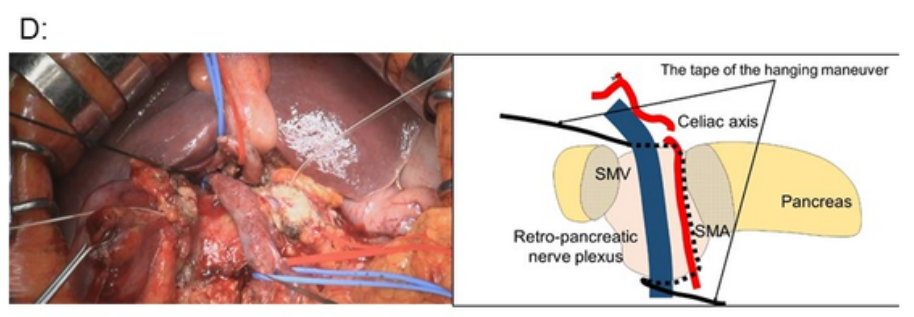

E:
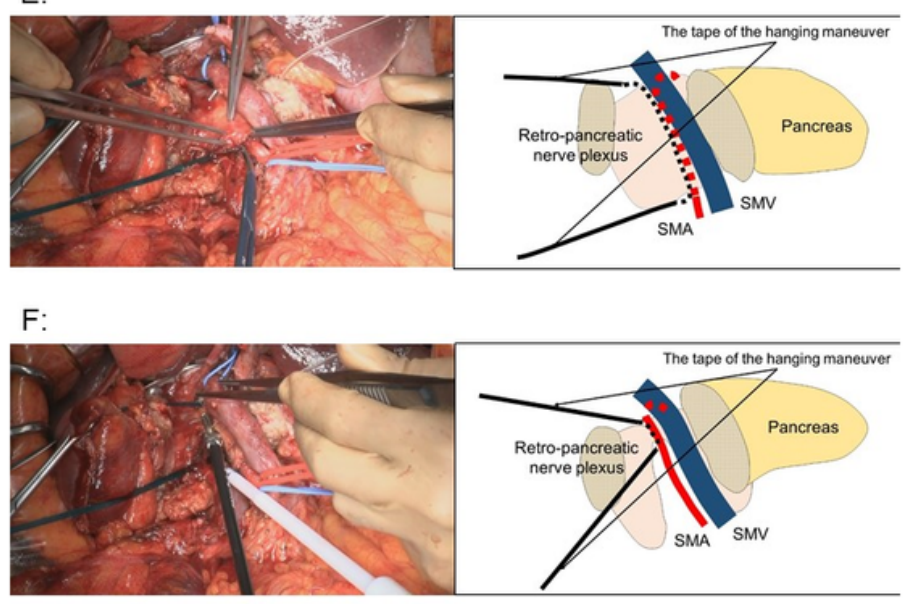

\section{Figure 1}

Picture and schema of pancreatoduodenectomy using the hanging maneuver of retropancreatic nerve plexus. A: After taping the SMA trunk from the anterior surface of the transverse mesocolon, the J1 artery and $\mathrm{J} 2$ artery were ligated and divided. After taping the SMV trunk, the J1 vein arising from the SMV was 
ligated and divided. The left part of the SMA in this procedure, which is the circle, is the exit of the hanging maneuver of the RNP. B: Dissection for kocherization was initiated at the outer edge of the duodenum and the pancreatic head was retracted to the left. The dissection layer was advanced to the left edge of the aorta to identify the root of the SMA and celiac axis. The cranial part of the celiac axis in this procedure was the entry of the hanging maneuver of the RNP, which is the circle. C: The entry of the hanging maneuver was connected to the dissection line on the right side of the celiac axis, which is the circle. D: After the dissection of the pancreatic parenchyma, the exit of the hanging maneuver could then be modified to subtract the SMA and SMV, and the entry could be modified to subtract the portal vein (PV). E: The tape of the hanging maneuver was pulled to the right side, and the RNP was deployed widely. F: While pulling the hanging tape, the RNP was easilly dissected using a sealing device. 


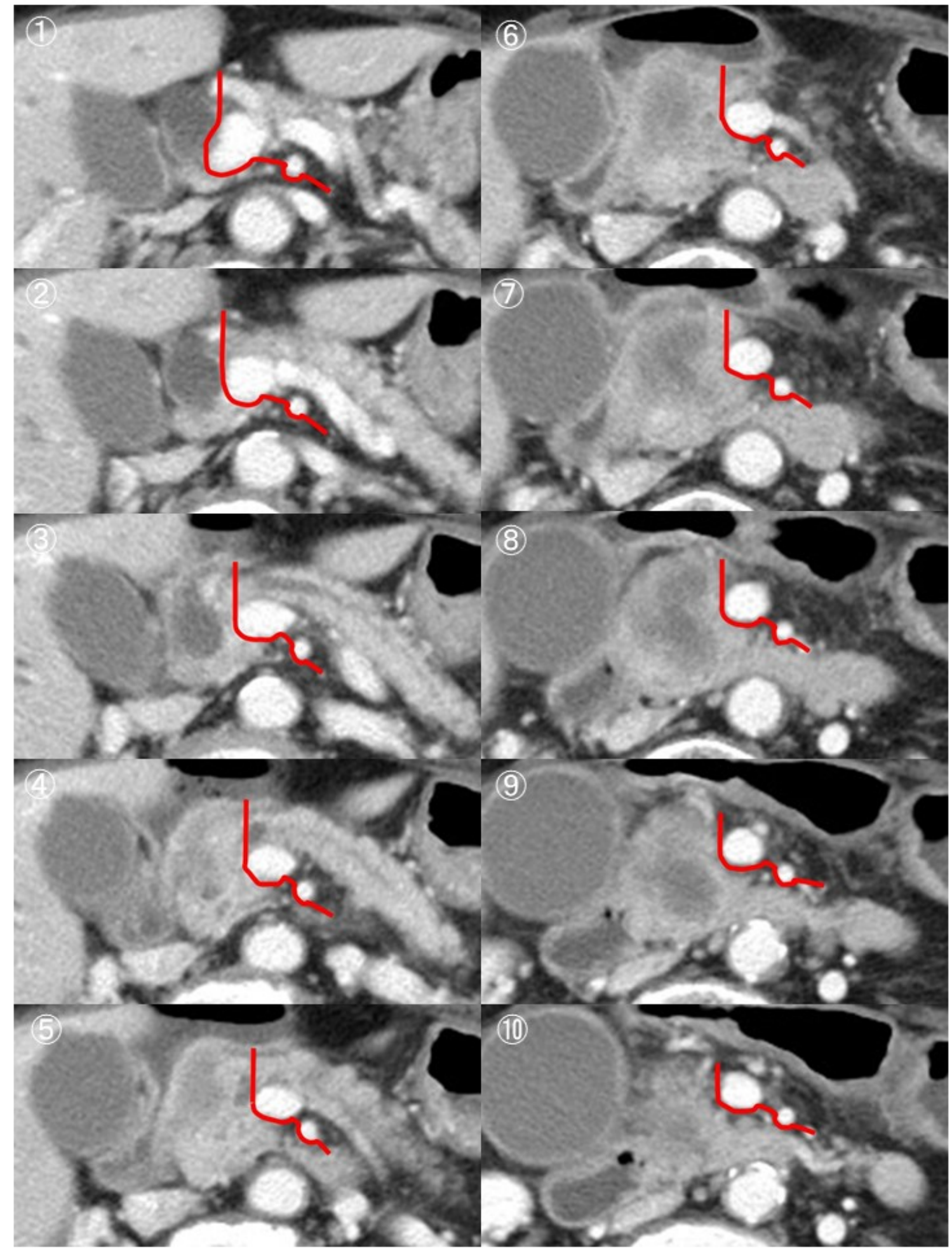

Figure 2

The oblique resection line along the SMA in computed tomography. Due to the anatomical features, the reliable excision line of the RNP always appears as an oblique line in computed tomography (CT) (red line). In the ventral approach, the cutting direction is diagonally inward unless the hanging maneuver is performed. 


\section{Supplementary Files}

This is a list of supplementary files associated with this preprint. Click to download.

- VideoformanuscriptPPPDHangingWMV.wmv 